\title{
Thiol Functionalization of Sesbania Gum and Its Evaluation for Mucoadhesive Sustained Drug Delivery
}

\author{
Shakuntla Verma ${ }^{1}$, Munish Ahuja ${ }^{*}$ \\ Drug Delivery Research Laboratory, Department of Pharmceutical Sciences, Guru Jambheshwar University of Science \\ and Technology, Hisar-125001(Haryana)
}

\begin{abstract}
The objective of present study was to improve muco-adhesiveness of sesbania gum by thiol functionalization. Thiolated sesbania gum was synthesized by reacting sesbania gum with mercaptoacetic acid in the presence of catalytic amount of acids. The modified gum was characterized physic-chemically and for biocompatibility. Thiolated sesbania gum was tested as mucoadhesive polymer for pharmaceutical applications by formulating its composite beads in the sodium alginate using metformin as a model drug. Thiolation onto sesbania gum was confirmed by Fourier transform infrared spectroscopy and energy dispersive X-ray -scanning electron micrographs. The degree of thiol substitution was found to be $1.72 \mathrm{mmol} / \mathrm{gm}$. The results of thrombogenic and haemolytic potential studies confirmed the biocompatibility of Thiolated sesbania gum. The comparative evaluation of composite beads of thiolated sesbania gum with sesbania gum and alginate alone beads revealed that thiolation of sesbania beads improves the bioadhesion property of sesbania gum.
\end{abstract}

Keywords: Sesbania gum, thiolation, bead, biocompatible, mucoadhesive

\section{INTRODUCTION}

A group of naturally occurring neutral polysaccharides i.e. galactomannans are most abundant raw material for industrial and pharmaceutical application due to easy availability, biodegradability, sustainability and non-toxic characteristics ${ }^{1}$.

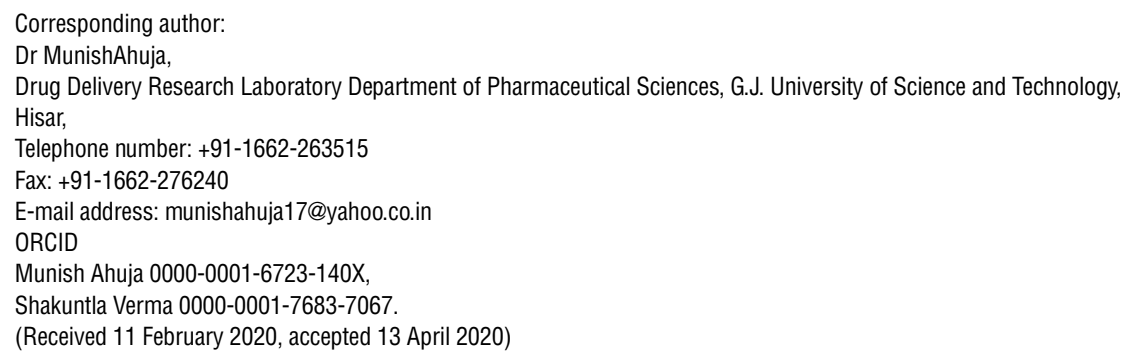


Galactomannans are composed of linear $\beta-(1 \rightarrow 4)$ glycosidic linked mannan backbone with $\alpha$-galactose side chain residues at C- 6 of mannose ${ }^{2}$. The various properties of galactomannans including molecular weight, mannose: galactose ratio (M:G) and attachment of galactose side chain residue on mannan backbone are believed to be responsible for their different rheological and physicochemical properties. Despite of various potential advantages of natural galactomannans, they owned certain limitations including less microbial stability and biodegradability. In order to overcome these problems and to enhance their utility, galactomannans are chemically modified via thiolation, carboxymethylation, microwave assisted grafting and many more. Thiolated polymers designated as thiomers havethiol group bearing side chain along the polymeric backbone ${ }^{3-5}$. These thiol groups are able to form disulfide linkage with mucosal glycoproteins resulting in higher mucoadhesiveness which further improve the therapeutic efficacy of the drug delivery system ${ }^{6}$. Numerous studies conducted earlier reported that thiolation modification of natural polysaccharide such as xanthan gum ${ }^{7}$, gellan gum ${ }^{8}$, pectin $^{9}$, tamarind seed polysaccharide ${ }^{10}$, Psyllium husk ${ }^{11}$, chitosan $^{12}$, alginate ${ }^{13}$ and hyaluronic acid ${ }^{14}$ improved their mucoadhesive properties.

Sesbania gum,a seed galactomannan, belongs to genus Sesbania and family Faboideae. The M:G ratio and average molecular weight of Sesbania gum is 2:1 and $2.3-3.4 \times 105 \mathrm{Da}$, respectively ${ }^{15}$. The gum form highly viscous suspension in aqueous system. Sesbania gum have been explored as diclofenac sodium loaded topical gelling agent ${ }^{16}$ and for colon targeting drug delivery of metronidazole ${ }^{17-18}$. Carboxymethyl functionalized sesbania gum has been evaluated as thickening agent in printing cotton fabrics with reactive dyes ${ }^{19}$. Also, the cross linking on sesbania gum using dialdehyde group was performed with changed thermal and swelling properties ${ }^{20}$; Sesbania gum was evaluated as filter-aid agent with enhancing leachability of rare-earth ore with ammonium sulphate solution as lixiviant ${ }^{21}$; Oxidized sesbania gum was formed using sodium hypochlorite and evaluated as wrap sizing agent for fine cotton yarns ${ }^{22}$; High adsorption capacity towards metal ions having epoxy functional groups into sesbania gum was evaluated $^{23}$. However, there are no reports on thiol modification of sesbania gum.

In the present investigation thiol functionalization of sesbania gum was carried out. The modified gum was characterized usingfourier transform infrared spectroscopy (FT-IR), scanning electron microscopy (SEM), energy dispersive X-ray micro-analysis (EDX) and thermo gravimetric analysis (TGA) studies. Thiolated sesbania gum was tested as mucoadhesive polymer by formulating composite beads with sodium alginate by ionic gelation method. The beads were evaluated for entrapment efficiency, in vitro release and mucoadhesive study. It was aimed 
to prepare metformin hydrochloride (model drug) loaded sesbania gum-sodium alginate (SG-Alg) and thiolated sesbania gum-sodium alginate (TSG-Alg) composite beads to achieve controlled drug delivery system.

\section{METHODOLOGY}

\section{Materials}

Sesbania gum and metformin hydrochloride were obtained as gift samples from Badar Enterprises (Jodhpur, Rajasthan, India). Mercaptoacetic acid, sodium alginate and methanol were purchased from Thomas Baker Chemicals Pvt. Limited (Mumbai, India). Sodium dihydrogen phosphate, hydrochloric acid and calcium chloridewere purchased from SD Fine-Chem Limited (Mumbai, India). Ellman's reagent [5,5'-dithiobis-(2-nitrobenzoic acid); $\mathrm{M}_{\mathrm{v}}=396.34 \mathrm{~g} / \mathrm{mol} ; 0.03 \% \mathrm{w} / \mathrm{v}$ ], di-sodium hydrogen phosphate, and L-Cysteine were purchased from HiMedia Laboratories Pvt. Ltd. (Mumbai, India).Freshly excised chick intestine was procured from local butcher house (Hisar, India).

\section{Synthesis of thiolated sesbania gum}

The synthesis of thiolated sesbania gum was done by esterification of native sesbania gum employing mercaptoacetic acid $(80 \%, w / v)$ in the presence of hydrochloric acid as catalyst. A dispersion of sesbania gum was prepared by adding 2 $\mathrm{g}$ of sesbania gum powder in $200 \mathrm{ml}$ cold distilled water with the aid of magnetic stirrer. The dispersion was reacted with mercaptoacetic acid (7.56 g) in the presence of $5 \mathrm{ml}$ of $7 \mathrm{~N} \mathrm{HCl}$ by refluxing at temperature of $7 \mathrm{O} \pm 2^{\circ} \mathrm{C}$ for $2 \mathrm{~h}$. The above reaction mixture was poured in $500 \mathrm{ml}$ methanol. White precipitate of thiolated sesbania gum so obtained was filtered using Whatman filter paper, washed with methanol and dried in oven at a temperature of $50 \pm 2^{\circ} \mathrm{C}^{24}$.

\section{Calculation of thiol group content}

The content of thiol group substitution in TSG was determined by wellestablishedEllman'smethod ${ }^{25}$. An aqueous dispersion (0.2\% w/v) of native Sesbania gum (control) and thiolated Sesbania gum was prepared. A volume of 2.5 $\mathrm{ml}$ of each prepared suspension was diluted with $2.5 \mathrm{ml}$ of phosphate buffer (0.5 $\mathrm{M}, \mathrm{pH}$ 8.0) followed by addition of $5 \mathrm{ml}$ of Ellman's reagent in it and allowed to react for $2 \mathrm{~h}$ at an ambient temperature in dark. Absorbance of the above reaction mixture was measured at $450 \mathrm{~nm}$ using UV spectrophotometer (UV-180o, Shimadzu, Japan). The number of thiol group substitution was calculated using calibration curve of $L$-cysteine (standard) with Ellman's reagent as detailed above. 


\section{Physiochemical characterization of sesbania gum and thiolated sesbania gum}

Both the gums i.e. native sesbania gum and thiolated sesbania gum were characterized for organoleptic and physical properties. Color, odour and taste like organoleptic characterization were done manually, while the physical properties such as density (bulk and tapped), angle of repose, Hausner's ratio, Carr's index and swelling index were calculated using standard procedures as follows-

\section{pH determination}

For $\mathrm{pH}$ determination of native and thiolated sesbania gum, a $2 \% \mathrm{w} / \mathrm{v}$ dispersion of each of the gum in distilled water was mixed with vigorous shaking for 10 $\min ^{26}$. The $\mathrm{pH}$ was measured using calibrated $\mathrm{pH}$ meter (Waterproof $p H T e s t r$ 10 , EUTECH instruments, OAKTON ${ }^{\circledR}$, Singapore).

\section{Bulk density and tapped density}

Accurately weighed amount of $5 \mathrm{gm}$ of sesbania/thiolated sesbania gum powder sample was introduced into $100 \mathrm{ml}$ measuring cylinder and the volume occupied by the powder was recorded as bulk volume. The measuring cylinder was tapped on a wooden frame till obtaining constant volume, which was taken as tapped volume ${ }^{27}$. The bulk density and tapped density was calculated as follows:

$$
\begin{gathered}
\text { Bulk } p=\frac{\text { Amonut of sample taken }}{\text { Bulk volume }} \\
\text { Tapped } p=\frac{\text { Amonut of sample taken }}{\text { Tapped volume }}
\end{gathered}
$$

\section{Angle of repose}

The angle between horizontal surface and apexof cone shaped pile of powder is characterized as the angle of repose. A glass funnel having the orifice diameter of $5 \mathrm{~cm}$ was fixed using a stand, $4 \mathrm{~cm}$ above the horizontal surface. The weighed amount of powder was then allowed to pass through the glass funnel followed by the measurement of diameter and height of the pile of powder ${ }^{27}$. The angle of repose was found using the equation:

$$
\tan \Theta=\frac{\text { Height of pile }}{\text { Diameter of pile }}
$$

Where, $\theta$ is the angle of repose 


\section{Hausner ratio and Carr's Index}

Hausnerratio and Carr's index provide flow properties and compressibility of powders. The values of bulk density and tapped density were used to determine these parameters using the formula:

$$
\begin{gathered}
\text { Hausner Ratio }=\frac{\rho_{\text {Tapped }}}{\rho_{\text {Bulk }}} \text { (4) } \\
\text { Carr's Index }=\frac{\rho_{\text {Tapped }}-\rho_{\text {Bulk }}}{\rho_{\text {Tapped }}} \text { (5) }
\end{gathered}
$$

\section{Swelling Index}

Swelling behaviour of sesbania/thiolated sesbania gum was determined by using modified method reported in previous literature ${ }^{28}$. A dispersion $(1 \% \mathrm{w} / \mathrm{v})$ of sesbania gum/thiolated sesbania gum powder was prepared and the initial (at 0 $\mathrm{h}$ ) and final volume (at $24 \mathrm{~h}$ ) occupied by the powder sediment was noted. The swelling index was determined using the given below equation:

Swelling Index $=\frac{V_{\text {Final }}-V_{\text {Initial }}}{V_{\text {Initial }}}$ X100 (6)

Where, $V_{\text {initial }}$ and $V_{\text {final }}$ are initial and final volume of powder, respectively

\section{Moisture content}

Moisture content affects the quality and stability of the product. However, it can be determined using thermogravimetric approach so, the moisture content of sesbania gum and chemically modified sesbania gum was calculated by evaporating them in a petridish (4g each) at $80^{\circ} \mathrm{C}$ in an oven until constant weight obtained ${ }^{29}$. The percentage moisture content was calculated via given formula:

Moisture content (\%)= $\frac{W_{\text {Initial }}-W_{\text {Final }}}{W_{\text {Initial }}}$ X100 (7)

Where, $\mathrm{W}_{\text {initial }}=$ Initial weight of sample and $\mathrm{W}_{\text {final }}=$ Final weight of sample after evaporation

\section{Fourier transformInfrared spectroscopy (FT-IR)}

Native sesbania gum and thiolated sesbania gum were subjected to spectrophotometer (IR-Affinity-1, Shimadzu, India)for functional properties confirmation at an ambient temperature using $\mathrm{KBr}$ pellets (Pellets were prepared by compress- 
ing the material for $30 \mathrm{sec}$ at the pressure of $75 \mathrm{~kg} / \mathrm{cm}^{2}$ in IR hydraulic press, CAP-15T, PCI Analytics, Mumbai, India) and scanned in the wave number range of $4000-400 \mathrm{~cm}^{-1}$.

\section{Thermal Analysis}

Thermogravimetric analysis study was conducted to investigate thermal stability and decomposition of sesbania gum and thiolated sesbania gum with the elevation of temperature. TGA analysis was performed employing Mettler Toledo TGA analyser (DSC3 PLUS, California, USA) in temperature ranging from $30-410^{\circ} \mathrm{C}$ under nitrogen atmosphere at a heating rate of $2{ }^{\circ} \mathrm{C}$ per minute.

\section{Scanning electron microscopy- Energy dispersive $X$-ray micro-anal- ysis (SEM-EDX)}

The size and surface morphological analysis of sesbania gum and thiolated sesbania gum were determined using scanning electron microscope (Apreo SEM, Thermo Scientific $\left.{ }^{\mathrm{TM}}\right)$. The specimens were coated with gold and mounted on specimen stubs using double adhesive carbon tape. Electron micrographs were captured at an accelerating voltage of $20 \mathrm{kV}$ at different magnifications. Presence of different element in native sesbania gum and thiolated sesbania gum were recorded using EDX image (AZtech,Oxford X $-\operatorname{Max}^{\mathrm{N}}$ ).

\section{Biocompatibility studies}

A comparative biocompatibility study of thiolated sesbania gum was performed against native sesbania gum for evaluation of its clot formation capability using thrombogenic and haemolytic potential. Gravimetric method was used to determine thrombogenic potential as discussed in previous literature ${ }^{30}$. The equal amounts of sesbania gum and thiolated sesbania gum (500 mg) was dispersed in phosphate buffer (20 ml, $\mathrm{pH}$ 7.2) for $24 \mathrm{~h}$ at room temperature. After complete hydration of both gums, samples were kept in whole citrated human blood (0.2 $\mathrm{ml}$ ) followed by mixing of $0.1 \mathrm{M} \mathrm{CaCl}_{2}(0.2 \mathrm{ml})$ and then distilled water (5 ml) after $45 \mathrm{~min}$. A volume of $5 \mathrm{ml}$ of formaldehyde (38\%) was added for fixing the clot formed which was dried further and weighed. The following equation was used to determine percentage thrombose:

$$
\text { Thrombose }=\frac{\text { Wt. of sample }- \text { Wt. of negative control }}{\text { Wt. of postive control }- \text { Wt. of negative control }} \text { X10o }
$$

Weight of positive control indicates weight of the clots without sample while weight of negative control represents weight of residue without blood and samples 
ASTM (American Society for Testing and Materials) standard was used to determine haemolytic potential as described in literature ${ }^{30}$. The same procedure was followed as mentioned above for hydration and clot formation of pure gum with its thiolated form. After incubating the samples in B.O.D. incubator (NSW- 152, Super Deluxe Automatic, India), centrifugation (Research centrifuge, TC 4100 D, Khera Instruments Pvt. Ltd., Delhi) was done at 10,000 rpm for $15 \mathrm{~min}$ for complete leaching of the unclotted blood. The absorbance of obtained supernatant fluid was analysed at $\lambda_{\text {max }}$ of $540 \mathrm{~nm}$ in UV Visible spectrophotometer (UV-180o, Shimadzu, Japan). The following equation was used to calculate the haemolytic index:

Haemolytic index $(\%)=$

Absorbance of sample - Absorbance of negative control

Absorbance of postive control - Absorbance of negative control $\mathrm{X} 100$

\section{Fabrication of drug loaded composite beads of thiolated sesbania gum with sodium alginate}

Composite beads of thiolated sesbania gum (TSG-Alg) and sesbania gum with sodium alginate (SG-Alg) were prepared by extrusion through a hypodermic needle in a crosslinking solution of calcium chloride. Previously, sesbania gum/ thiolated sesbania gum (500mg) and sodium alginate (500mg) was dispersed in $0.1 \mathrm{~N} \mathrm{NaOH}(20 \mathrm{ml})$ and deionized water, respectively. Both the suspensions i.e. sesbania gum/thiolated sesbania gum and sodium alginate were mixed followed by addition of model drug i.e. metformin hydrochloride (150mg) with continuous stirring. To obtain the adequate composite beads, the prepared suspensions were dropped into $\mathrm{CaCl}_{2}$ solution (30 ml) using hypodermic needle (24 \#) from a height of $26 \mathrm{~cm}$ over the period of $2 \mathrm{~min}$, and the beads formed were kept for 10 min so that cross-linking could take place $^{31}$. On completion of cross-linking reaction, obtained composite beads were filtered, taken out and dried in petridish at ambient temperature. For comparison purpose, metformin hydrochloride loaded sodium alginate beads (Alg) were also prepared using above mentioned procedure. 


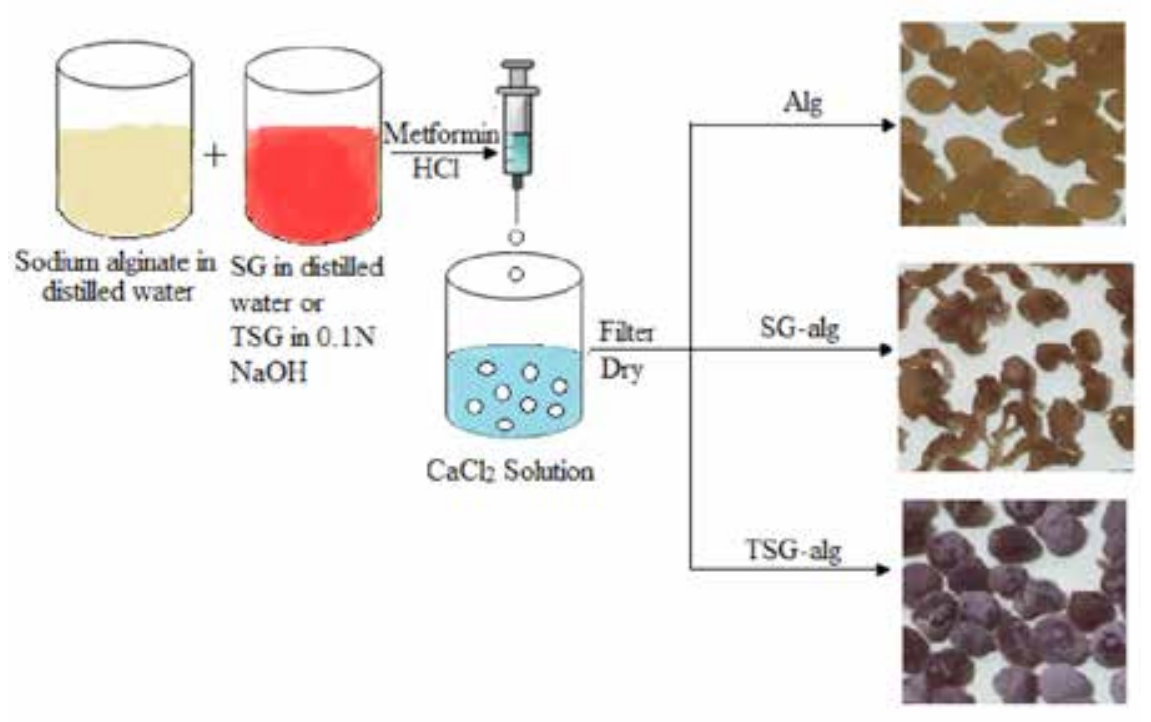

Figure 1: Schematic representation of bead formulation.

\section{Characterization of drug loaded composite beads}

The beads of various batches so obtained were evaluated for percentage yield, percentage entrapment efficiency, swelling behaviour, Fourier-transform infrared spectroscopy, scanning electron microscopy and in-vitro release behaviour.

\section{Percentage yield}

The yield (\%) of SG-Alg,TSG-AlgandAlgwere calculated by using the given formula:

Yield (\%)= $\frac{\mathrm{Wt}_{\text {Beads }}}{\mathrm{Wt}_{\text {Polymer+Meffor.HCl }}}$ X100 (10)

Where,

$\mathrm{Wt}_{\text {Beads }}=$ Total weight of beads produced

$\mathrm{Wt}_{\text {Polymer+Metfor.HCl }_{\mathrm{HCl}}}=$ Total weight of sesbania gum/thiolatedsesbania gum/sodium alginate and drug used in the formulation of beads

\section{Entrapment efficiency}

The amounts of drug in gum included or not included in the beads were evaluated spectrophotometrically.Briefly, about $100 \mathrm{mg}$ of crushed beads from each batch i.e. composite beads and sodium alginate beads were digested in $50 \mathrm{ml}$ phosphate 
buffer ( $\mathrm{pH}$ 6.8) using probe sonication (Q55, QSonica, USA) for 5 min (amplitude $40 \%$ ). Aliquots from the filtrate, remaining after filtration of polymer debris were assayed using a spectrophotometer at $234 \mathrm{~nm}$ (UV 1800, Shimadzu, Japan).The amount of entrapped metformin hydrochloride was calculated as follows:

Entrapment efficiency (\%)= $\frac{\text { Metfor. } H C l_{\text {Practical }}}{\text { Metfor. } H C l_{\text {Theoritical }}}$ X100 (11)

Where,

Metfor. $\mathrm{HCl}_{\text {Practical }}=$ amount of metformin hydrochloride found in beads

Metfor. $\mathrm{HCl}_{\text {Theoritical }}=$ amount of metformin hydrochloride calculated to be present in thebeads

\section{In-vitro release study}

The release of metformin hydrochloride from SG-Alg, TSG-Alg and Alg beads were performed using USP dissolution test apparatus (Paddle Type, TDL-o8L, Electrolab, Mumbai, India) in $900 \mathrm{ml}$ dissolution medium (0.2 M phosphate buffer, $\mathrm{pH}$ 6.8). The beads having metformin hydrochloride equivalent to $100 \mathrm{mg}$ were tied in muslin cloth and suspended under the paddle ${ }^{32}$. Then the paddle was immersed in phosphate buffer solution for $24 \mathrm{~h}$ at $50 \mathrm{rpm}$ and the temperature was maintained at $\left(37 \pm 0.5^{\circ} \mathrm{C}\right.$. At predetermined interval of time, aliquots of $5 \mathrm{ml}$ sample were removed and replaced by the same volume of fresh dissolution medium to maintain the sink condition during the whole test. The withdrawn samples were filtered using syringe filter $(0.45 \mu \mathrm{m})$ and analysed using UV-Visible spectrophotometer (UV 1800, Shimadzu, Japan) at $234 \mathrm{~nm}$.

\section{Swelling study}

The swelling behaviour of SG-Alg, TSG-Alg and Alg beads were determined in solutions of different $\mathrm{pH}(1.2,6.8$ and 7.2$)$ at $(37 \pm 0.5)^{\circ} \mathrm{C}$ up to $24 \mathrm{~h}$. Briefly, about $100 \mathrm{mg}$ of beads from each batch was kept in $200 \mathrm{ml}$ buffer solution ${ }^{31}$. Weight of swollen beads after blotting the excess liquid adhered on the surface was recorded at different interval of time until constant weight. The \% swelling was calculated using the given formula:

Swelling (\%)= $\frac{\mathrm{Wt}_{\mathrm{s}}-\mathrm{Wt}_{\mathrm{D}_{\mathrm{D}}}}{\mathrm{Wt}_{\mathrm{D}}} \mathrm{X} 100(12)$

Where, Wt. ${ }_{\mathrm{s}}$ and Wt. ${ }_{\mathrm{D}}$ are the weights of swollen and dry beads, respectively. 


\section{Mucoadhesive study}

Mucoadhesivestudy of SG-Alg, TSG-Alg and Alg beads were carried out by wash off method using freshly excised chick intestine purchased from local butcher house (Hisar, India) ${ }^{9}$. Adipose and connective tissue of the isolated intestine was removed properly. Then, tissue was adhered with mucosal surface (facing outside) on glass slide using cyanoacrylate glue. About 150 beads of each batch were adhered by pressing lightly on mucosal surface. The prepared glass slide was hung into the beaker having phosphate buffer solution (pH 6.8) on the USP tablet disintegration test machine ${ }^{33}$ for $24 \mathrm{~h}$. Total number of beads detached was noted at specific time interval. The whole study was carried out in triplicate manner.

\section{RESULTS AND DISCUSSION}
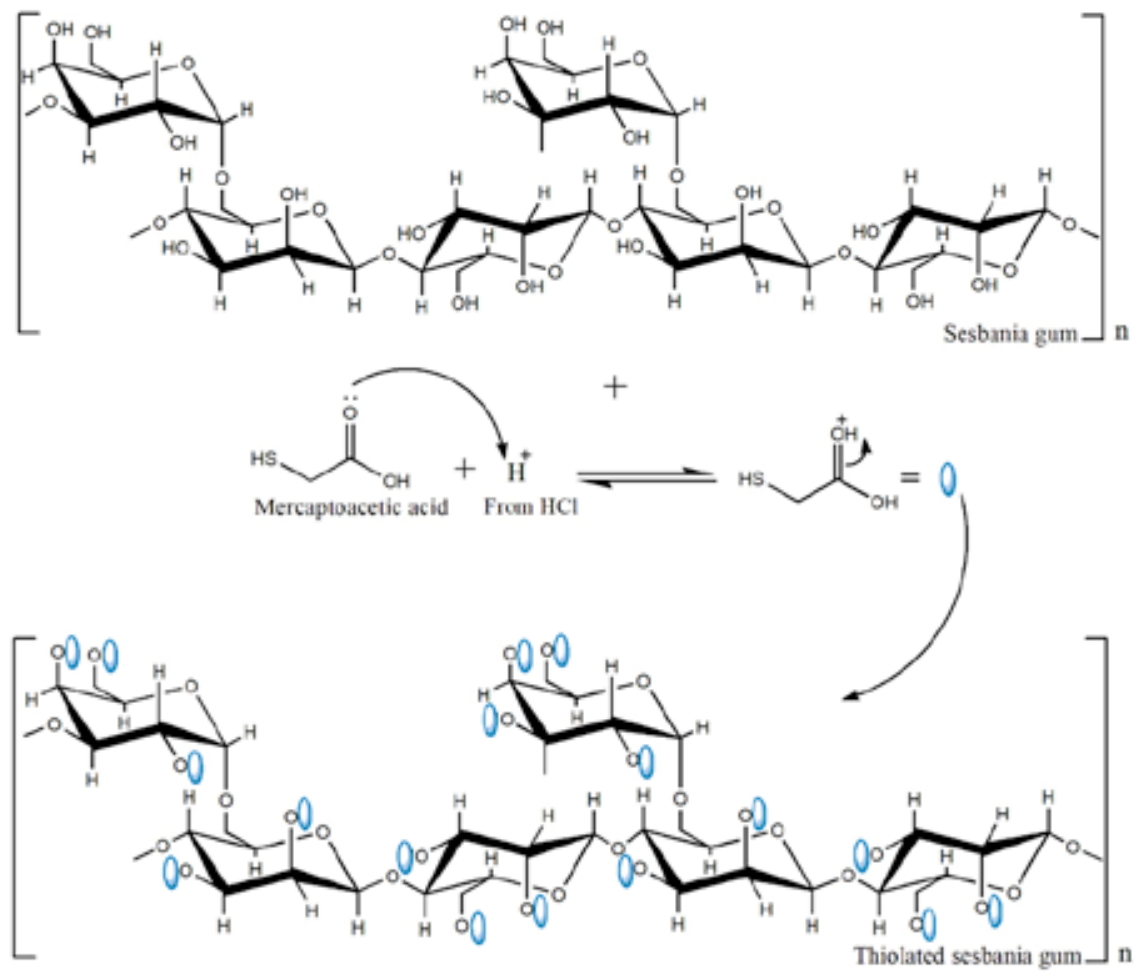

Figure 2: Schematic diagram for thiolation of sesbania gum.

Sesbania gum, a galactomannan has been chemically modified by employing mercaptoacetic acid under acidic conditions for thiol derivatization as shown in figure 2. In the first step of thiolation process, the hydroxyl groups (-OH) exhibited on sesbania gum are substituted by chlorine which further react with the carboxyl 
groups (-COOH) of mercaptoacetic acid having sulfhydryl (-SH) present at terminal to form thiolated sesbania gum ${ }^{3}$. The air dried reaction product so obtained was copper red in colour with the characteristic odour. It was soluble in alkaline medium. The \% yield of modified sesbania gum was found to be $89.65 \%$. The number of thiol groups substitution was found to be $1.72 \mathrm{mmol} / \mathrm{gm}$, which was determined by Ellman's method.

The physicochemical properties of sesbania gum were considerably altered after thiolation. An aqueous dispersion (2.5\% w/v) of thiolatedsesbania gum was found to be more acidic than the sesbania gum. The $\mathrm{pH}$ of sesbania gum gets decreased after thiolation as shown in table 1. Similar results were earlier reported for thiolated starch, which were attributed to the higher affinity of thiol group for accepting electron pair and donation of $\mathrm{H}^{+}$ions ${ }^{29}$. The bulk and tapped densities after chemical modification of sesbania gum were increased. The Hausner ratio and Carr's index were calculated from the bulk and tapped density. The Hausner ratio is indicator of interparticulate friction. The lower values of Hausner ratio on thiolation of sesbania gum points towards lesser friction among the thiolatedsesbania gum particles as compared to the sesbania gum ${ }^{27}$. Further the Carr's Index which refers to the bridge strength and stability of powder was also found to diminish on thiol functionalization. The angle of repose which is traditionally used to characterize the flow properties of powders also shows a decrease in value on thiolation. On the basis of the result of Hausner ratio, Carr's Index and angle of repose measurements, it can be concluded that the sesbania gum can be characterized as passable to very poor flow properties powder, whereas thiolated sesbania gum powder exhibit fair to good flow behaviour.

Table 1. Different parameters of sesbania and thiolated sesbania gum

\begin{tabular}{ccc}
\hline Parameters & Sesbania gum & Thiolatedsesbania gum \\
\hline $\mathrm{pH}$ & $6.2 \pm 0.1$ & $2.9 \pm 0.1$ \\
Bulk density $\left(\mathrm{g} / \mathrm{cm}^{3}\right)$ & $0.51 \pm 0.02$ & $0.81 \pm 0.02$ \\
Tapped Density $\left(\mathrm{g} / \mathrm{cm}^{3}\right)$ & $0.75 \pm 0.01$ & $1 \pm 0.01$ \\
Angle of repose $\left({ }^{\circ}\right)$ & $42.26 \pm 0.5$ & $21.8 \pm 0.7$ \\
Hausner's ratio & $1.49 \pm 0.01$ & $1.22 \pm 0.01$ \\
Carr's index & $32.65 \pm 0.03$ & $19 \pm 0.04$ \\
Swelling $(\%)$ & $30 \pm 1.24$ & $10 \pm 1.11$ \\
Moisture content $(\%)$ & $7.7 \pm 0.6$ & $2.8 \pm 0.4$ \\
\hline
\end{tabular}

Data are presented as mean $\pm \mathrm{SD}(\mathrm{n}=3)$ 
In terms of swelling, thiolated sesbania gum has found less swelling power than pure sesbania gum as shown in table 1 . It can beexplained by the fact that the native sesbania gumhas large numbers of hydroxyl groups which form intermolecular hydrogen bond with water molecules while in case of thiolated sesbania gum,SH groups present in thiolated sesbania gumform weak H-bonds as compared to $-\mathrm{OH}$ groups because the thiol groups have less polarity and dipole moment as compared to the corresponding alcohols ${ }^{34}$.

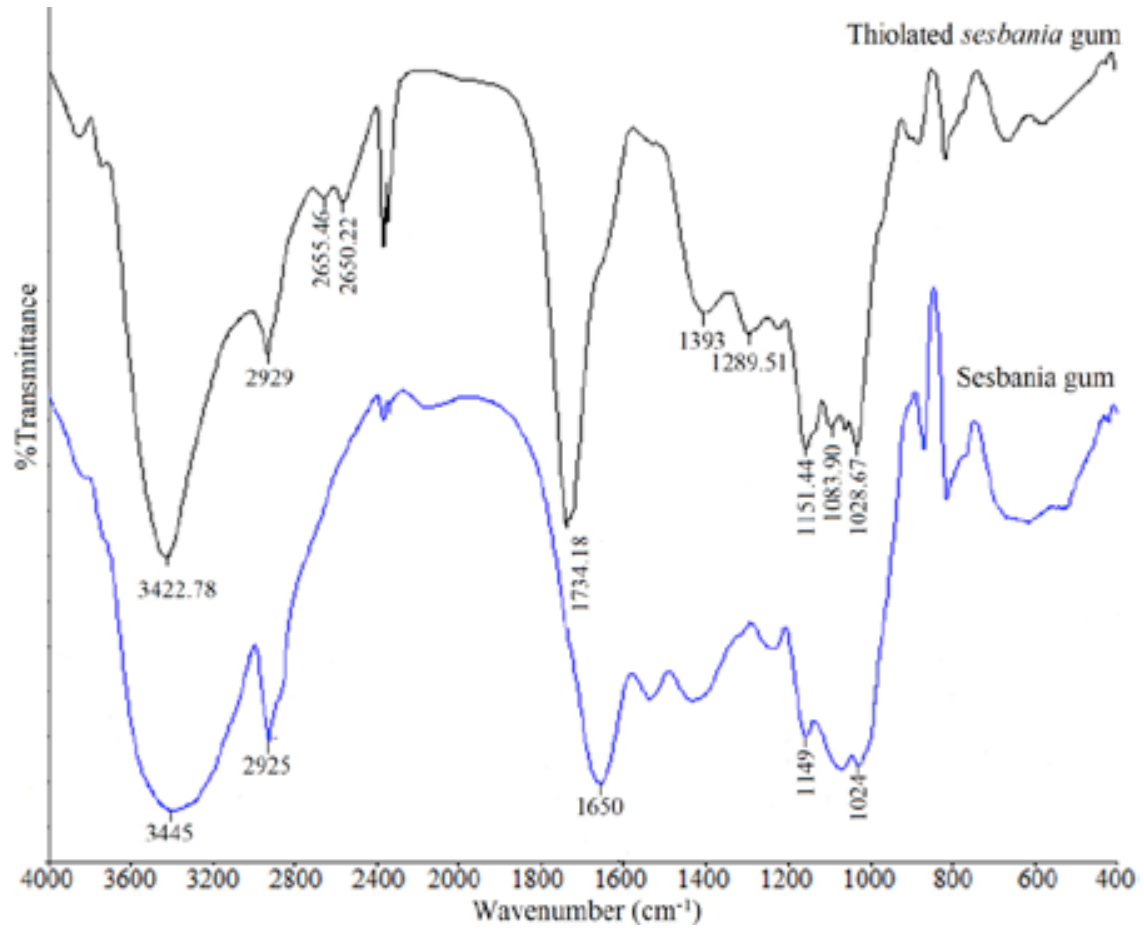

Figure 3: FT-IR spectrum of thiolated sesbania gum and sesbania gum.

Figure 3 represent FT-IR spectra of sesbania and thiolated sesbania gum. The broad and strong absorption band in native sesbania gum which appears at $3445 \mathrm{~cm}^{-1}$ is due to $\mathrm{O}-\mathrm{H}$ group stretching. Thepresence of $\mathrm{C}-\mathrm{H}$ linkage of alkane is shown at $2925 \mathrm{~cm}^{-1}$, while the peak at $1650 \mathrm{~cm}^{-1}$ is attributed to $\mathrm{C}=\mathrm{O}$ stretching of primary alcohols and two small peaks at $1149 \mathrm{~cm}^{-1}$ and $1024 \mathrm{~cm}^{-1}$ are due to $\mathrm{C}=\mathrm{O}$ stretching of tertiary alcohol ${ }^{22}$. FTIR spectrum of thiolated sesbania gum showed a narrow band at $3442.78 \mathrm{~cm}^{-1}$ which is due to free $\mathrm{O}-\mathrm{H}$ group stretching of mercaptoacetic acid, while the presence of $\mathrm{C}-\mathrm{H}$ linkage of alkane is shown at 2929 $\mathrm{cm}^{-1}$; C-H bending at $1734.18 \mathrm{~cm}^{-1} ; \mathrm{O}-\mathrm{H}$ bending at $1393 \mathrm{~cm}^{-1} ; \mathrm{C}=\mathrm{O}$ stretching at $1289.51 \mathrm{~cm}^{-1}$ and $1028.67 \mathrm{~cm}^{-1} ; \mathrm{C}=\mathrm{O}$ stretching of primary and tertiary alcohol at 
$1083.90 \mathrm{~cm}^{-1}$ and $1151.44 \mathrm{~cm}^{-1}$, respectively; small peak at $2655.46 \mathrm{~cm}^{-1}$ is related to $\mathrm{O}-\mathrm{H}$ stretching;one extra stretch at $2650.22 \mathrm{~cm}^{-1}$ is due to $-\mathrm{SH}$ stretching of thiol group which confirms thiolation of sesbania gum. Thiol bands are not easy to detect using FT-IR spectroscopy; therefore, various other studies were performed.
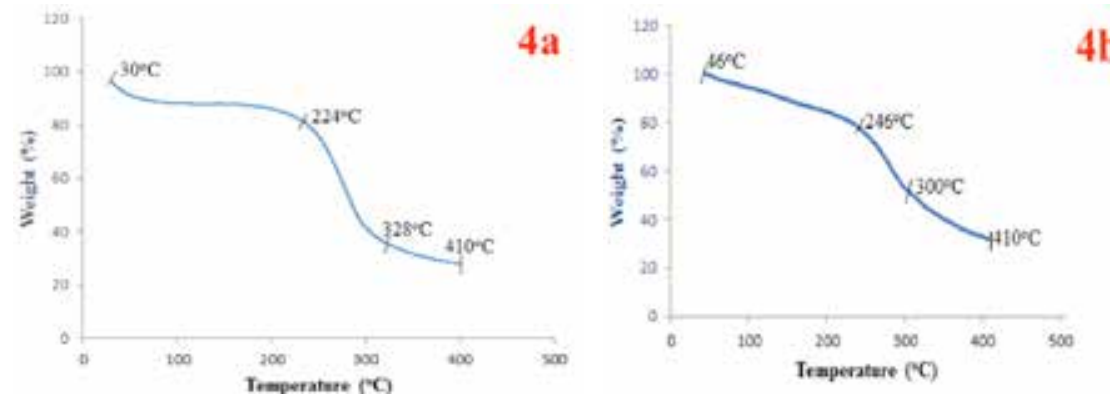

Figure 4: TGA plot of sesbania and modified thiolated sesbania gum.

Figure 4 exhibits thermal plots of sesbania and thiolated sesbania gum showing weight loss (\%) with temperature while reviewing the thermogram, it was observed that decomposition occurs in three successive phases showing sigmoid curve. In native sesbania gum, first stage of decomposition occurs from $30^{\circ} \mathrm{C}$ to $224^{\circ} \mathrm{C}$ with $14 \%$ weight loss while $48 \%$ weight loss occurred during second stage in temperature range of $225^{\circ} \mathrm{C}-328^{\circ} \mathrm{C}$. In third stage of thermal degradation from $329^{\circ} \mathrm{C}-410^{\circ} \mathrm{C}, 8 \%$ loss of weight was observed. In thiolated sesbania gum, first stage of decomposition was from $46^{\circ} \mathrm{C}$ to $246^{\circ} \mathrm{C}$ in which $24 \%$ weight loss occurred. In second stage of degradation which occurred between $247^{\circ} \mathrm{C}$ and $300^{\circ} \mathrm{C}$, $24 \%$ weight loss occurred while in third stage $\left(301^{\circ} \mathrm{C}-410^{\circ} \mathrm{C}\right), 21 \%$ weight loss occured. At the end of the thermal study at $410^{\circ} \mathrm{C}$, a residue of $30 \%$ of Sesbania gum and $31 \%$ of thiolated sesbania gum was left which indicate that there is no difference between thermal stability of sesbania gum and thiolated sesbania gum ${ }^{24}$. The weight loss during first stage of degradation is due to desorption of bound water and dehydration because of loss of hydroxyl groups from the polysaccharide backbone ${ }^{35}$, while the weight loss during the second and third stages can be ascribed to depolymerisation and the pyrolysis resulting in the evolution of gaseous products such as carbon monoxide, carbon dioxide, methane etc ${ }^{36}$. 

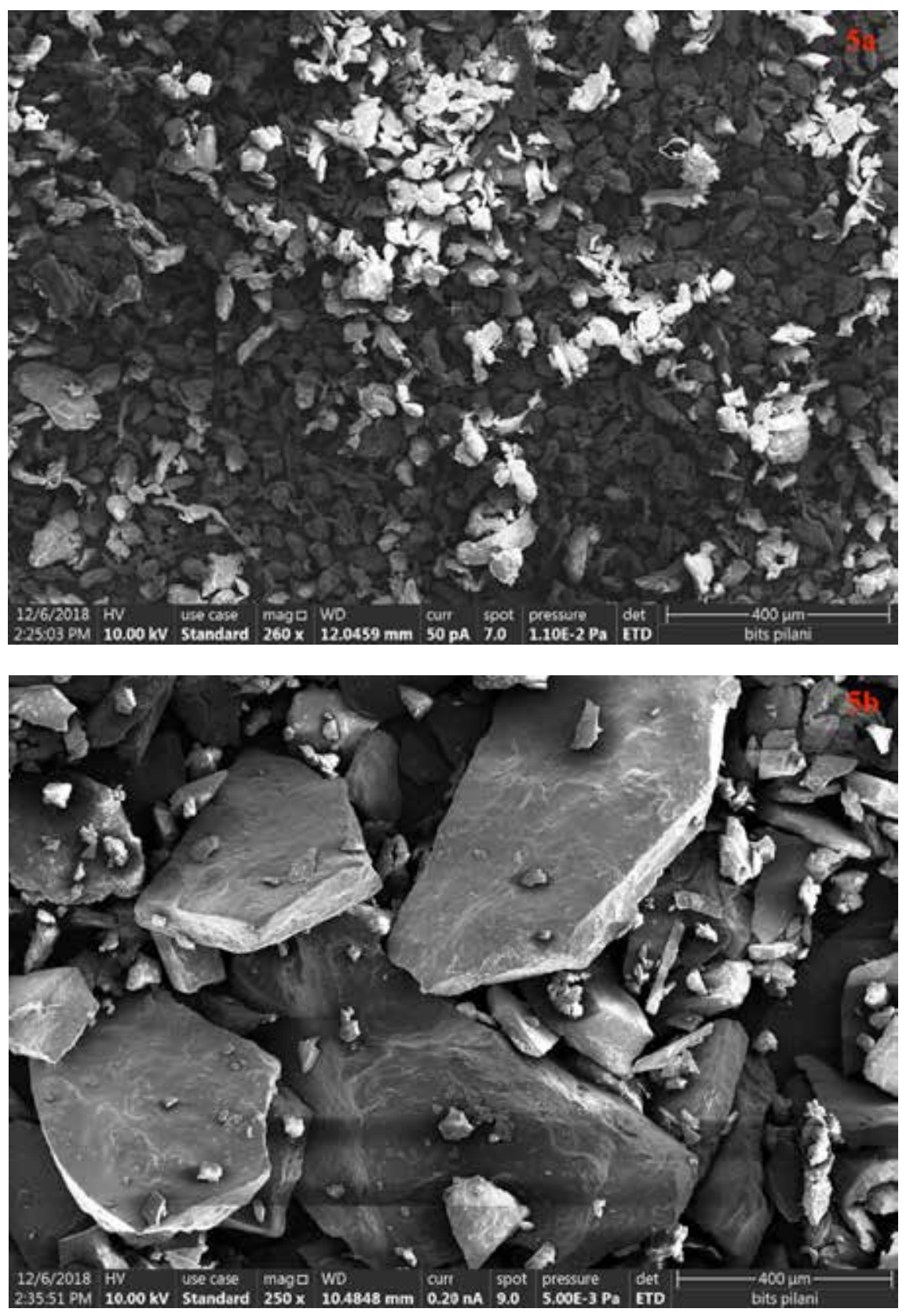

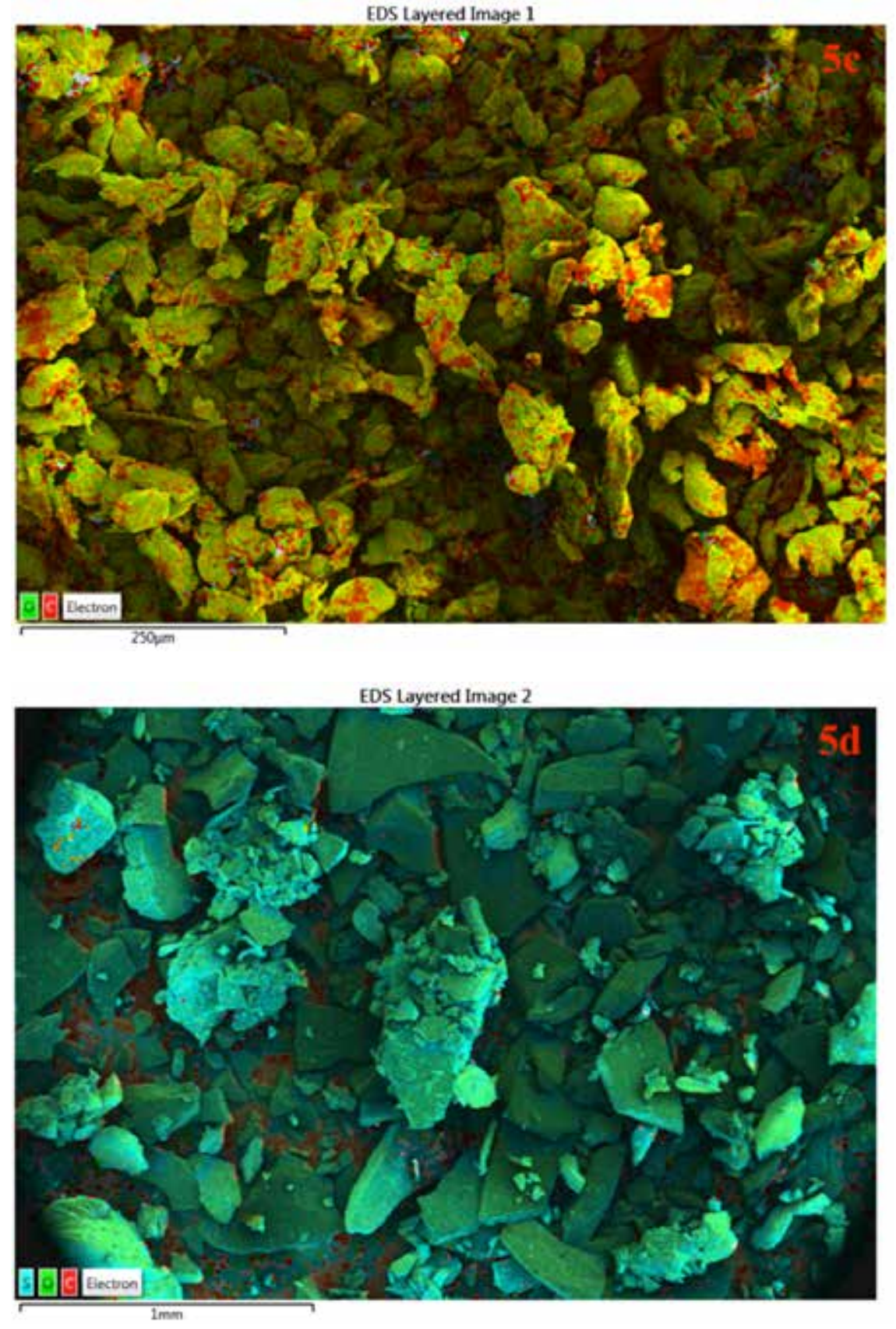

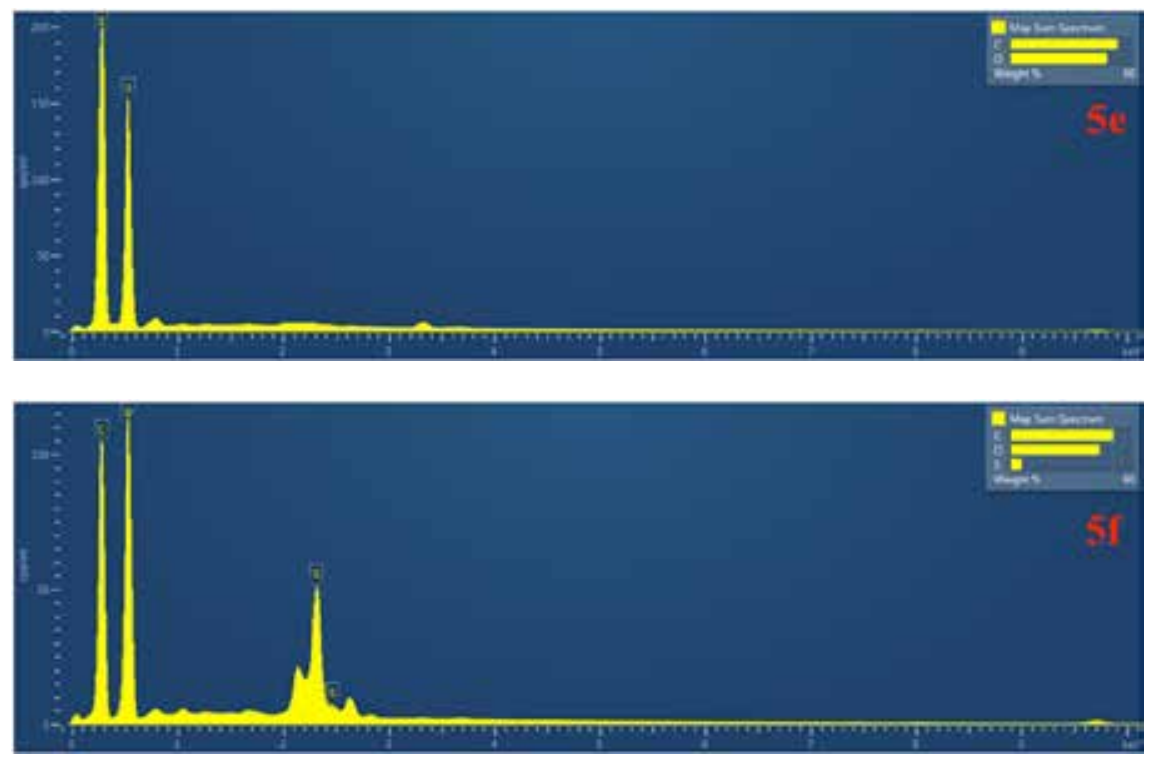

Figure 5: Field emission-scanning electron micrographs - Energy dispersive $X$-ray analysis (FE-SEM-EDX) of sesbania gum (a, c, e) and modified sesbania gum (b, d, f).

Scanning electron micrographs of sesbania and thiolated sesbania gum have been shown in figure 5 . As clearly shown in photographs of sesbania gum (fig. 5 a), polyhedral flakes are present while SEM micrographs of thiolated sesbania gum (fig 5b), show presence of plate shape particles. Fig 5 shows the EDX layered images of sesbania (c) and thiolated sesbania gum (d). The presence of carbon is indicated by red color while green color represents oxygen in figure $5(\mathrm{c})$. In the EDX layered image of thiolated sesbania gum (fig. 5 d), additional blue color shows the presence of sulphur. Further, the EDX spectrum of thiolated sesbania gum (fig. 5f) also shows the additional peak of sulphur at $2.3 \mathrm{keV}$, which confirms the presence of sulphur in thiolated sesbania gum. This peak is not present in the EDX spectrum of sesbania gum (fig $5 \mathrm{e}$ ).

Biocompatibility study was performed on native sesbania and thiolated sesbania gum for analysis of its clot formation capability. The test revealed that clot formation in native sesbania gum ( $0.132 \mathrm{~g}$ in $2 \mathrm{ml}$ citrated whole blood) and thiolatedsesbania gum ( $0.157 \mathrm{~g}$ in $2 \mathrm{ml}$ citrated whole blood) was less than as compared to positive control clot weight ( $0.181 \mathrm{~g}$ in $2 \mathrm{ml}$ of citrated whole blood). The \% thrombose of sesbania and thiolated sesbania gum was calculated i.e. $71 \%$ for sesbania gum and $85 \%$ for thiolated sesbania gum which concludes that both sesbania and thiolated sesbania gum can be non-thrombogenic. Further, hemolytic index of sesbania and thiolated sesbania gum was found to be $2.85 \%$ and $1.30 \%$ respectively which is also considered as safe and suitable for drug delivery applications ${ }^{30}$. 
Thiolated sesbania gum was further tested for drug delivery applications by using it as a mucoadhesive polymer. Since thiolated sesbania gum as such does not form ionically gelled beads, it was used in combination with sodium alginate to prepare composite beads using metformin hydrochloride as a model drug and $\mathrm{CaCl}_{2}$ as a cross-linking agent. For comparative study, composite beads of sesbania gum with sodium alginate and the beads of sodium alginate alone were also prepared. The composite beads of thiolated sesbania and alginate beads (TSG$\mathrm{Alg}$ ), sesbania gum and sodium alginate (SG-Alg) and alginate alone beads (Alg) were obtained in a percentage yield of $122.3 \%, 141.2 \%$ and $97.4 \%$, respectively. The entrapment efficiency of TSG-Alg, SG-Alg and Alg beads was found to be $99.96 \%, 99.26 \%$ and $89.03 \%$, respectively.

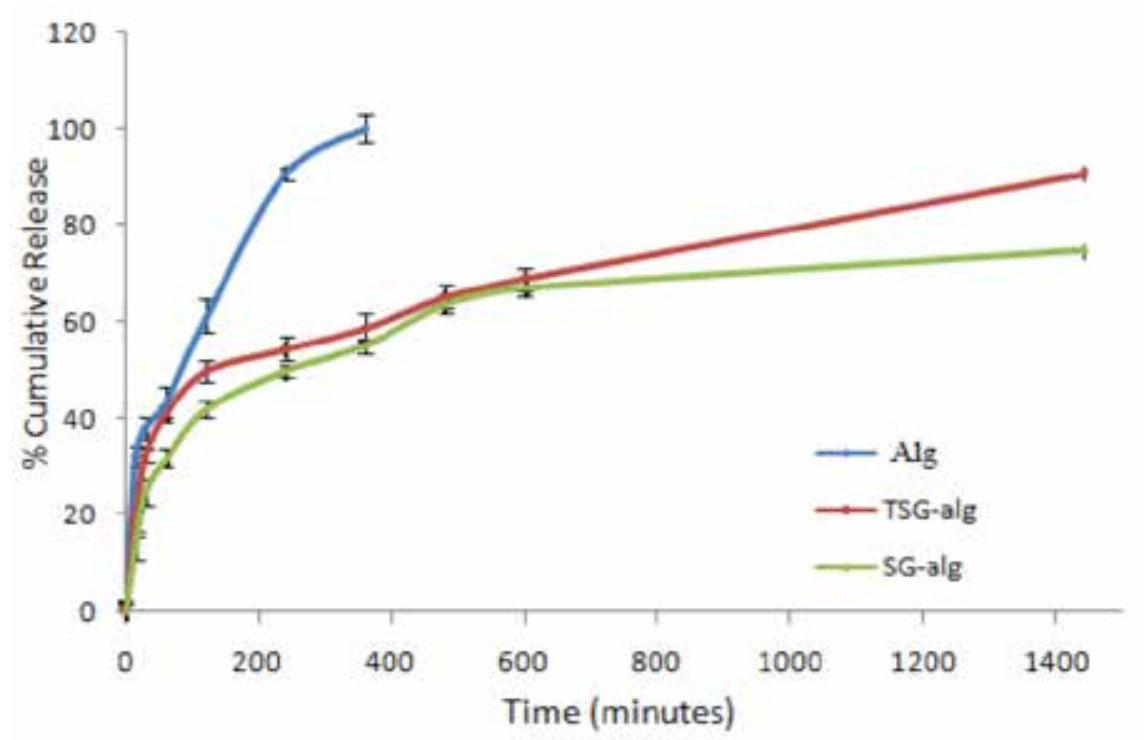

Figure 6: In-vitro drug release of Alg, SG-Alg and TSG-Alg composite beads.

Figure 6 represents the comparative in-vitro release profile of metformin from the Alg, TSG-Alg and SG-Alg composite beads. The drug release behaviour was studied in phosphate buffer (pH 6.8) to evaluate its release kinetics. It can be seen in the figure thatTSG-Alg composite beads released $90.68 \%$ of drug in 24 $\mathrm{h}$ whereas SG-Alg composite beads released $74.85 \%$ of metformin in the same time. As compared to composite beads, Alg beads released almost $100 \%$ of the drug in $6 \mathrm{~h}$. This indicates that composite beads of sesbania gum/thiolatedsesbania gumwith alginate are more effective in sustaining the release of metformin as compared to the beads of alginate alone. It can be observed from the profile 
that almost similar pattern of release is observed for the release of metformin from composite beads of SG-Alg and TSG-Alg. Only at $24^{\text {th }} \mathrm{h}$ there was somewhat greater difference in the release of metformin from the TSG-Alg beads. Further to check whether there is any significant difference in the release of metformin from the two composite beads, the release data was evaluated for determining $f_{1}$ and $f_{2}$ value. On comparing the release data of metformin from TSG-Alg and SG-Alg beads, the $f_{1}$ and $f_{2}$ value were found to be 13.07 and 55.58 , respectively which indicates that the release profile of metformin from the two beads can be considered to be similar. Further the release rate data was fitted into various kinetic models for determining the release kinetic and mechanism of release (Table 2). The results revealed that the release of metformin from the alginate (Alg) and composite beads of sesbania gum and alginate beads (SG-Alg) follows Higuchi square-root kinetics while in case of composite beads of thiolated sesbania gum ad alginate (TSG-Alg), the release data fits best into $1^{\text {st }}$ order kinetics. The value of ' $n$ ' the release exponent of Korsemeyer and Peppas equation $(n<0.45)$ which indicates that the release of metformin from all batches of the beads occurs primarily by diffusion through matrix.

Table 2 Modelling and release kinetics of TSG-Alg,SG-Algand Alg.

\begin{tabular}{crrccc}
\hline & & \multicolumn{2}{c}{$\mathbf{R}^{2}$} & \multirow{2}{*}{ 'n' } \\
\cline { 2 - 5 } Formulation & Zero order & lstorder & Higuchi square-root & $\begin{array}{l}\text { Korseme- } \\
\text { yer-Pep- } \\
\text { pas }\end{array}$ & \\
\hline TSG-Alg & 0.693 & 0.950 & 0.908 & 0.900 & 0.330 \\
SG-Alg & 0.626 & 0.782 & 0.887 & 0.937 & 0.421 \\
Alg & 0.871 & 0.966 & 0.981 & 0.969 & 0.300 \\
\hline
\end{tabular}

Table 3 lists the results of swelling behaviour and ex-vivo bioadhesion study of TSG-Alg, SG-Alg composite beads and alginate beads in phosphate buffer $\mathrm{pH}$ 6.8).The results of swelling study support the release rate profile. The Alg beads dissolved within $6 \mathrm{~h}$ releasing almost all the drug. On the other hand, SG-Alg beads continued to swell till 24h sustaining the release of metformin, while TSGAlg beads continued to swell till $12 \mathrm{~h}$ and then started to erode thereby releasing drug at slightly faster rate than the SG-Alg beads. 
Table 3. Swelling behaviour and Ex-vivobioadhesion study of Alg, SG-Alg and TSG-Alg

\begin{tabular}{|c|c|c|c|c|c|c|}
\hline \multirow{2}{*}{ Time (h) } & \multicolumn{3}{|c|}{ Swelling (\%) } & \multicolumn{3}{c|}{ Ex-vivobioadhesion time (\%) } \\
\cline { 2 - 7 } & Alg & SG-Alg & TSG-Alg & Alg & SG-Alg & TSG-Alg \\
\hline $\mathbf{0 . 5}$ & $45.21 \pm 1.23$ & $77.41 \pm 2.55$ & $20 \pm 1.32$ & 100 & 100 & 100 \\
\hline $\mathbf{1}$ & $223.48 \pm 1.44$ & $183.81 \pm 2.12$ & $153.33 \pm 1.22$ & 100 & 100 & 100 \\
\hline $\mathbf{2}$ & $486.67 \pm 2.13$ & $294.23 \pm 2.34$ & $320.34 \pm 1.12$ & 95.4 & 100 & 100 \\
\hline $\mathbf{4}$ & $256.45 \pm 1.47$ & $483.87 \pm 2.17$ & $520 \pm 1.25$ & 50.9 & 100 & 100 \\
\hline $\mathbf{6}$ & $21.11 \pm 1.69$ & $516.13 \pm 1.99$ & $706.67 \pm 1.09$ & 30 & 86.6 & 100 \\
\hline $\mathbf{1 2}$ & - & $541.93 \pm 1.33$ & $893.33 \pm 1.11$ & 28 & 80 & 100 \\
\hline $\mathbf{2 4}$ & - & $554.83 \pm 1.76$ & $320 \pm 2.05$ & 5 & 73 & 93.3 \\
\hline
\end{tabular}

The mucoadhesive ability of the metformin loaded beads of the different batches of the beads was evaluated comparatively by determining bioadhesion (table 3). It can be observed from the results that Alg beads could adhere to the intestinal mucosal tissue only upto 6h. On the other hand, composite beads of SG-Alg shows $100 \%$ adhesion till $4 \mathrm{~h}$ and at the end of $24 \mathrm{~h}$ of the study $73 \%$ of the beads still adhered to the intestinal mucosal tissue. In case of composite beads of TSG-Alg, $100 \%$ of the beads were found adhering till $12 \mathrm{~h}$ and at the end of $24 \mathrm{~h}$ study period $93.3 \%$ of beads were found adhering to the intestinal mucosal tissue. The results thus conform the higher mucoadhesivity of TSG-Alg as compared to the SG-Alg beads. The literature already reported that sulfhydryl group (-SH) present in thiolated polymers form strong covalent disulphide bond with glycoproteins present in mucus which was clearly indicated in its results also ${ }^{33}$. However, hydroxyl group (-OH) present in sesbania gum form weak hydrogen bond or show weak Van der Waal's interaction with mucus glycol-proteins and show less mucoadhesionproperty ${ }^{37}$. Similar results were earlier observed in thiolated pectin $^{9}$ and thiolated alginate beads ${ }^{38}$.

This study introduces the thiolation modification on sesbania gum with their characterization. The sesbania gum was esterified using mercaptoacetic acid to formulate thiolated sesbania gum which was characterized physiochemically, structurally, morphologically, and thermally. Thiolated sesbania gum was also found biocompatible as compared to native sesbania gum in the biocompatibility study. Fabrication of metformin drug loaded composite beads with sodium alginate i.e. Alg, SG-Alg and TSG-Alg beads were done with characterization. The thiolated sesbania gum shows $90.68 \%$ in-vitro drug release following $1^{\text {st }}$ order kinetics. Thiol group on sesbania gum enhance the mucoadhesive strength which can also be explored for pharmaceutical applications. 


\section{REFERENCES}

1. Wu, Y.; Li, W.; Cui, W.; Eskin, N. A. M.; Goff, H. D. A molecular modeling approach to understand conformation-functionality relationships of galactomannans with different mannose/ galactose ratios. Food Hydrocoll. 2012, 26, 359-364.

2. Srivastava, M.; Kapoor, V. P. Seed Galactomannans: An Overview. Chem. Biodivers. 2oo5, 2, 295-317.

3. Leichner, C.; Jelkmann, M.; Bernkop-Schnurch, A. Thiolated polymers: Bioinspired polymers utilizing one of the most important bridging structures in nature. Adv. drug deliv. Rev. 2o19, 151-152, 191-221.

4. Gok, K.M.; Demir, K.; Cevher, E.; Ozsoy, Y.; Cirit, U.; Basinoglu, S.; Ozgumus, S.; Pabuccuoglu. S. The effects of the thiolation with thioglycolic acid and l-cysteine on the mucoadhesion properties of the starch-graft-poly (acrylic acid). Carbohydr. Polym. 2017, 163, 129-136.

5. Bernkop-Schnurch, A. Thiomers: a new generation of mucoadhesive polymers. Adv. Drug Deliv. Rev. 2005, 57, 1569-1582.

6. Kulkarni, D. A.; Joshi, A. A.; Patil, L. C.; Amale, D. P.; Patel, M. H.; Surana, J. S.; Belgamwar, S. V.; Chaudhari, S. K.; Pardeshi, V. C. Xyloglucan: A functional biomacromolecule for drug delivery applications. Int. J. Biol. Macromol. 2017, 104, 799-812.

7. Bhatia, M.; Ahuja, M.; Mehta, H. Thiol derivatization of xanthan gum and its evaluation as a mucoadhesive polymer. Carbohydr. Polym. 2015, 131, 119-124.

8. Yadav, S.; Ahuja, M.; Kumar, A.; Kaur, H. Gellan-thioglycolic acid conjugate: Synthesis, characterization and evaluation as mucoadhesive polymer. Carbohydr. Polym. 2014, 99, 601-607.

9. Sharma, R.; Ahuja, M. Thiolated pectin: Synthesis, characterization and evaluation as a mucoadhesive polymer. Carbohydr. Polym. 2o11, 85, 658-663

10. Kaur, H.; Yadav, S.; Ahuja, M.; Dilbaghi, N. Synthesis, characterization and evaluation of thiolated tamarind seed polysaccharide as a mucoadhesive polymer. Carbohydr. Polym. 2o12, 90, 1543-1549.

11. Bhatia, M.; Ahuja, M. Thiol modification of psyllium husk mucilage and evaluation of its mucoadhesive applications. Sci. world J. 2013, 1-7.

12. Mahmood, A.; Lanthaler, M.; Laffleur, F.; Huck, W. C.; Bernkop-Schnurch, A. Thiolated chitosan micelles: Highly mucoadhesive drug carriers. Carbohydr. Polym. 2017, 167, 250-258.

13. Hauptstein, S.; Bernkop-Schnurch, A. Synthesis and in vitro characterization of a novel Sprocted thiolated alginate. Carbohydr. Polym. 2015, 124, 1-7.

14. Kafedjiiski, K.; Bernkop-Schnurch, A. Synthesis and in vitro evaluation of thiolated hyaluronic acid for mucoadhesive drug delivery. Int. J. Pharm. 2007, 343, 48-58.

15. Ma, X.; Pawlik, M. Effect of alkali metal cations on adsorption of guar gum onto quartz.Colloid Interface Sci. 2005, 289, 48-55.

16. Patel, G. C.; Patel, M. M. Preliminary Evaluation of sesbania seed gum mucilage as gelling agent. Int. J. Pharm. Tech. Res. 2009, 1, 840-843.

17. Patel, G. N.; Patel, R. B.; Patel, H. R. Formulation and in-vitro evaluation of microbially triggered colon specific drug delivery using sesbania gum. $e-J$. Sci. Technol. 2o11, 6, 33-45.

18. D. Chandra, A. P. Singh, P.K, Singh, J. K. Maurya, T. Raj, Am. J. Pharm. Tech. Res. 2o13, 3 409-426.

19. Rekaby, M. M.; El-Thalouth, A. I.; Rahman, H. A. A. El-Khabery El-Satar A. S. Technological 
evaluation of carboxymethyl sesbania galactomannan gum derivatives as thickeners in reactive printing. Bio. Resources. 2010, 5, 1517-1529.

20. Hongbo, T.; Shiqi, G.; Yanping, L.; Siqing, D. Modification mechanism of sesbania gum, and preparation, property, adsorption of dialdehyde cross-linked sesbania gum. Carbohyd. Polym. 2016, 149, 151-162.

21. Tian, J.; Tang, X.; Yin, J.; Chen, J.; Luo, X.; Rao, G. Enhanced leachability of a lean weathered crust elution-deposited rare earth ore: effects of sesbania gum filter-aid reagent. Metall. Mater. Trans. B. 2013, 44B, 1070-1077.

22. Shen, D.; Xue, M.; Zhang, L.; Liu, H.; Gao, L.; Cui, Y. Preparation and characterization of oxidized sesbania gum and evaluation of its warp sizing performance for fine cotton yarns. Polym. Degrad. Stabil. 2o11, 96, 2181-2188.

23. Zhang, Q.; Gao, Y.; Zhai, A. Y.; Liu, Q. F.; Gao, G. Synthesis of sesbania gum supported dithiocarbamate chelating resin and studies on its adsorption performance for metal ions. Carbohydr. Polym. 2oos, 73, 359-363.

24. Grewal, P.; Mundlia, J.; Ahuja, M. Thiol modified Moringa gum-A potential bioadhesive polymer. Carbohydr. Polym. 2019, 209, 400-408.

25. Bernkop-Schnurch, A., Hornof, M., \& Zoidl, T. Thiolated polymers-thiomers: synthesis and invitro evaluation of chitosan-2-iminothiolane conjugates. Int. J. Pharm. 2oo3, 260, 229-237.

26. Falade, O. K.; Okafor, A. C. Physical, functional, and pasting properties of flours from corms of two Cocoyam (Colocasia esculenta and Xanthosoma sagittifolium) cultivars. J. Food Sci. Technol. 2015, 52, 3440-3448.

27. Shah, B.R.; Tawakkul, A.M.; Khan, A.M. Comparative Evaluation of Flow for Pharmaceutical Powders and Granules. AAPS Pharm. Sci. Tech. 2oo8, 9, 250-258.

28. Nagpal, M.; Aggarwal, G.; Jain, K. U.; Madan, J. Extraction of gum from Abelmoschus esculentus physicochemical peculiarity and antioxidant prepatent. Asian J. Pharm. Clin. Res. 2o17, 10, 174-179.

29. Das, S.; Das, K.M. Synthesis and characterization of thiolated jackfruit seed starch as a colonic drug delivery carrier. Int. J. Applied Pharm. 2o19, 11, 53-62.

30. Singh, B.; Kumar, A. Network formation of Moringa oleifera gum by radiation induced crosslinking: Evaluation of drug delivery, network parameters and biome-dical properties. Int. J. Biol. Macromol. 2018, 108, 477-488.

31. Nayak, K.A.; Pal, D.; Pradhan, J.; Hasnain, S.M. Fenugreek seed mucilage-alginate mucoadhesive beads of metformin $\mathrm{HCl}$ : design, optimization and evaluation. Int. J. Biol. Macromol. 2013, 54, 144-154.

32. Verma, S.; Ahuja, M. Carboxymethyl sesbania gum: synthesis, characterization and evaluation for drug delivery. Int. J. Biol. Macromol. 2017, 98, 75-83.

33. Ahuja, M.; Singh, S.; Kumar, A. Evaluation of carboxymethyl gellan gum as a mucoadhesive polymer. Int. J. Biol. Macromol. 2013, 53, 114-121.

34. Cleaves, H. J., II, Thiol, in Encyclopedia of Astrobiology, M. Gargaud et al., eds., p. 1668, Springer, New York, 2011.

35. Piao, J.; Lee, J. E.; Weon, K. Y.; Kim, D. W.; Lee, J. S.; Park, J. D. S.; Development of novel mucoadhesive pellets of metformin hydrochloride. Arch. Pharm. Res. 2oo9, 32, 391-397.

36. Zohuriaan, M. J.; Shokrolahi, F. Thermal studies on natural and modified gums. Polym.

Test. 2oo4, 23, 575-579. 
37. Bahulkar, S. S.; Munot, M. N.; Surwase, S. S. Synthesis, characterization of thiolated karaya gum and evaluation of effect of $\mathrm{pH}$ on its mucoadhesive and sustained release properties. Carbohydr. Poly. 2015, 130, 183-19o.

38. Kassem, A. A.; El-zamarany, E. A. Development of mucoadhesive microbeads using thiolated sodium alginate for intrapocket delivery of resveratrol. Int. J. Biol. Macromol. 2015, 487, 305313 . 